\title{
Rationale and options for combination therapy in the treatment of Type 2 diabetes
}

\author{
L. F. Van Gaal ${ }^{1,2}$, I. H. De Leeuw ${ }^{2}$ \\ ${ }^{1}$ University Hospital of Antwerp, Faculty of Medicine, Department of Diabetology, Metabolism and Clinical Nutrition, \\ Edegem (Antwerp), Belgium \\ 2 Department of Diabetology, Metabolism and Clinical Nutrition, University Hospital Antwerp, Antwerp - Edegem, Belgium
}

\begin{abstract}
Pharmacological therapy for Type 2 (non-insulin-dependent) diabetes mellitus aims at controlling hyperglycaemia to delay or prevent complications associated with the disease. Most patients with Type 2 diabetes present with both stimulated insulin deficiency and insulin resistance. In general, the former can manifest as postprandial hyperglycaemia and the latter as fasting hyperglycaemia, though a definitive association has not been established. Emerging data show a high failure rate of long-term monotherapy and establishes the significance of mealtime glycaemia and the role of postprandial glucose excursions in the development and progression of vascular complications. To overcome such failures of monotherapy and to address the different underlying defects of the pathology of Type 2 diabetes, a combined therapy of oral antidiabetic agents with complementary modes of action should be considered. Currently used oral antidiabetic agents such as sulphonylureas, biguanides (metformin)
\end{abstract}

and the thiazolidinediones (rosiglitazone, pioglitazone) commonly target fasting hyperglycaemia and have limited additive effects on postprandial glycaemia. In contrast, $\alpha$-glucosidase inhibitors can reduce postprandial hyperglycaemia but gastrointestinal side effects restrict their use. The development of new agents to control postprandial glucose excursions could be considered as an additional objective for the management of Type 2 diabetes. To this end new short-acting enhancers of insulin secretion such as repaglinide (benzoic acid derivative) and nateglinide (amino acid derivative) have been developed. The combination of such agents with other complementary modes of action, e.g. an insulin sensitizer, could target better major underlying defects of Type 2 diabetes and thereby provide a better approach for controlling the entire glycaemic risk. [Diabetologia (2003) 46[Suppl]:M44-M50]

Keywords Type 2 diabetes, postprandial glucose, nateglinide, D-phenylalanine derivative, insulin-secretion enhancer, combination therapy.
Guidelines for the management of Type 2 (non-insulindependent) diabetes mellitus have recently been modified to take into account the need to attain near normal

Received: 27 September 2001 / Revised: 18 June 2001

Published online: 5 February 2003

C Springer-Verlag 2003

Corresponding author: L. F. Van Gaal, University Hospital of Antwerp, Faculty of Medicine, Department of Diabetology, Metabolism and Clinical Nutrition, Wilrijkstraat 10, 2650 Edegem (Antwerp), Belgium

E-mail: luc.van.gaal@uza.be

Abbreviations: UKPDS, United Kingdom Prospective Diabetes Study; PPAR- $\gamma$, peroxisome proliferator-activated receptor- $\gamma$. glycaemia to reduce the risk of microvascular and macrovascular complications. The current guidelines for glycaemic control in Type 2 diabetes set aggressive targets of $\mathrm{HbA}_{1 \mathrm{c}}$ of less than $7 \%$ or even less than $6.5 \%[1,2]$. Such challenging targets suggest that clinicians need to re-evaluate their treatment strategies to attain near-normal $\mathrm{HbA}_{1 \mathrm{c}}$ values. For example, good glycaemic control $\left(\mathrm{HbA}_{1 \mathrm{c}}<7 \%\right)$ has been achieved by only about $25 \%$ of patients treated with metformin monotherapy with a mean baseline $\mathrm{HbA}_{1 \mathrm{c}}$ of 8.2 to $8.4 \%[3,4]$. These data, and those from other clinical studies, suggest that combination therapy may be required to reach glycaemic control targets in patients who have early Type 2 diabetes (i.e. $\mathrm{HbA}_{1 \mathrm{c}} \leq 9 \%$ ). 
The introduction of multiple classes of oral antidiabetic agents that act differentially to correct the metabolic defects which characterise Type 2 diabetes has greatly expanded the potential for combination therapy. An understanding of the metabolic pathogenesis of diabetes and the pharmacological mechanisms of action of antidiabetic drugs can facilitate their rational use.

Both impaired beta-cell function and increased insulin resistance occur in patients with IGT and in those with Type 2 diabetes. In IGT and early Type 2 diabetes the early phase of insulin secretion is lost, mainly resulting in the characteristic increased postprandial glucose. Increased insulin resistance also frequently occurs in people who are obese and is associated with the metabolic syndrome [3].

The traditional focus of antidiabetic agents was mainly to reduce fasting plasma glucose (FPG) but convincing evidence now exists showing the importance of controlling both postprandial and fasting hyperglycaemia $[1,2,3,4]$. Postprandial hyperglycaemia, when the multiple homeostatic mechanisms that minimise glucose excursions and restore euglycaemia after a meal are blunted, is one of the earliest abnormalities of glucose homeostasis in patients with IGT and Type 2 diabetes. In these patients, the early phase of insulin secretion in response to a glucose challenge is lost and, although overall insulin exposure is not altered, increases in postprandial blood glucose are higher, longer and have a greater variability than in non-diabetic subjects [2, 4]. Furthermore, because a considerable amount of time is spent in the non-fasted state, mealtime glucose excursions make a considerable contribution to overall glycaemic control [4]. If postprandial hyperglycaemia is not adequately controlled it can worsen insulin resistance and further impair insulin secretory capacity. As the early phase of insulin secretion 'primes' insulin-sensitive tissues and promotes the uptake of glucose, its restoration in patients with Type 2 diabetes is important for the control of mealtime glycaemia [5].

The overall aim of pharmacological therapy is to control hyperglycaemia and to delay or avert microvascular and possibly macrovascular complications by maintaining long-term euglycaemia. The link between tight control of fasting glycaemia and a reduction in microvascular complications was first established in patients with Type 1 diabetes [6] and subsequently confirmed in Type 2 diabetic patients [7, 8, 9]. Although most studies did not measure postprandial glycaemia, glycosylated haemoglobin $\left(\mathrm{HbA}_{1 \mathrm{c}}\right)$ was found to be a strong predictor of major cardiovascular events. The equal contribution made by postprandial and fasting glycaemia to $\mathrm{HbA}_{1 \mathrm{c}}$ indirectly confirms the importance of both fasting and prandial glycaemia in the prevention of complications, particularly in the early stages of the disease. Furthermore, postprandial hyperglycaemia has recently been shown to be an independent risk factor for the development of macrovascular complications $[4,10,11]$. In the Diabetes Intervention Study, intensive glucose control was associated with a lower incidence of coronary heart disease (CHD) whereas postprandial, but not fasting, hyperglycaemia was an independent risk factor for death [12]. More recently, it has been shown that $\mathrm{HbA}_{1 \mathrm{c}}$ concentrations predict mortality, with risk increasing throughout the entire range of $\mathrm{HbA}_{1 \mathrm{c}}$ concentrations, even below the threshold commonly accepted for the diagnosis of diabetes [13].

\section{Combination therapy for Type 2 diabetes}

Dietary and lifestyle measures are fundamental for managing Type 2 diabetes. Nevertheless, apart from the stage of diabetes at diagnosis, large numbers of patients require pharmacological combination therapy to achieve good glycaemic control $\left(\mathrm{HbA}_{1 \mathrm{c}}<7 \%\right)$ [14]. Furthermore, because Type 2 diabetes is a progressive disease, even patients with good initial responses to monotherapy will eventually require combination treatment $[7,15,16,17]$. The rational use of oral combination therapy can delay the need for multiple insulin injections, facilitates the maintenance of low $\mathrm{HbA}_{1 \mathrm{c}}$ and helps prevent vascular complications.

Oral antidiabetic agents can be divided into two broad categories according to their mechanism of action: those that enhance insulin secretion and those that target insulin resistance [18] (Table 1). Within the

Table 1. Classification and mode of action of oral antidiabetic agents with potential for use in combination therapy

\begin{tabular}{llll}
\hline Mode of action & Class & Example & Primary control \\
\hline Augment insulin supply & Sulphonylureas & Glyburide & Fasting plasma glucose \\
& Benzoic acid derivatives & Repaglinide & Postprandial glucose \\
& Amino acid derivatives & Nateglinide & Postprandial glucose \\
Reduce insulin resistance & Biguanides & Metformin & Fasting plasma glucose \\
& Thiazolidinediones & Rosiglitazone & Fasting plasma glucose \\
Reduce intestinal breakdown & $\alpha$-glucosidase inhibitors & Acarbose & Postprandial glucose \\
of complex carbohydrates & & &
\end{tabular}




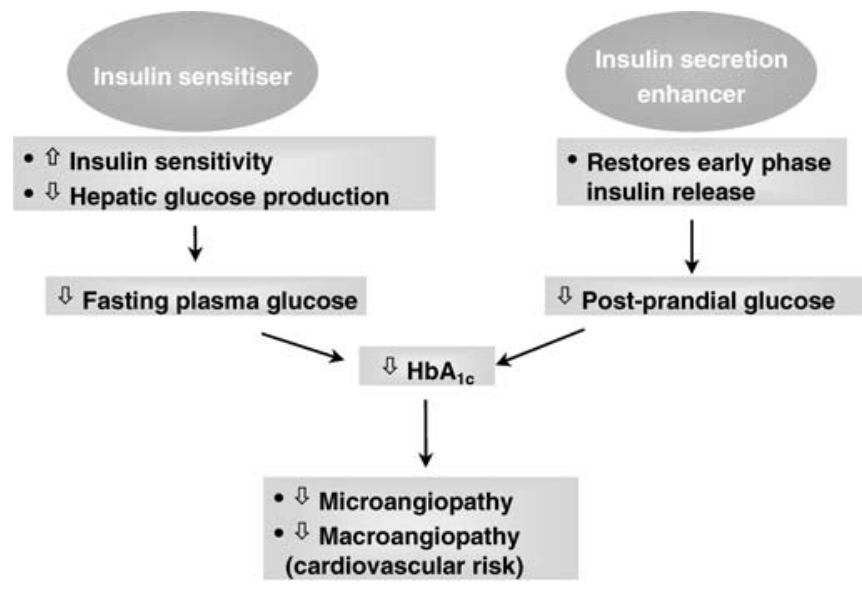

Fig. 1. Classification of antidiabetic agents according to their mechanism of action

latter category, this effect can be achieved by increasing insulin sensitivity (e.g. thiazolidinediones, biguanides), reducing hepatic glucose production (biguanides), and delaying intestinal glucose absorption ( $\alpha$ glucosidase inhibitors). Because most patients with Type 2 diabetes have both impaired insulin secretion and increased insulin resistance, a rational approach is to use different classes of agents to ameliorate each defect (Fig. 1) [18, 19].

\section{Combination regimens currently in use}

Combination therapy has focused mainly on adding insulin-sensitizing medications to the sulfonylureas, the oldest and most widely used hypoglycaemic drugs. Sulphonylureas, previously the mainstay of treatment, provided optimal efficacy and few side effects at low dosages but, apart from their tendency to cause hypoglycaemia, can become less effective over time due to the progression of the disease (reduced insulin secretory reserve), which has led to their use in combination with insulin-sensitizers, including metformin and, more recently, thiazolidinediones. In the United Kingdom Prospective Diabetes Study (UKPDS), addition of metformin to a sulphonylurea increased the proportion of patients achieving an $\mathrm{HbA}_{1 \mathrm{c}}$ of less than $7 \%$ at 3 years: $33 \%$ in the sulphonylurea plus metformin group compared with $21 \%$ with sulphonylurea alone $\left(p=0.007\right.$; baseline $\mathrm{HbA}_{1 \mathrm{c}}$ 7.4 and $7.3 \%$, respectively) [15]. Also in a Swedish study, a combination therapy of glibenclamide and metformin at low doses, started relatively early after diagnosis, led to successful metabolic control with fewer side effects than on either of the respective monotherapies [20].

Several clinical trials have shown these agents to have additive effects and no more adverse effects together than alone. The use of an $\alpha$-glucosidase inhibitor in combination with sulphonylureas, metformin or insulin also results in improved glycaemic control [21, $22,23,24]$. These agents inhibit the hydrolysis of complex carbohydrates in the gastrointestinal tract and thereby delay postprandial glucose absorption. Provided that the patients' food contains a sufficient amount of complex carbohydrates, overall $\alpha$-glucosidase inhibitors are less effective in lowering FPG than sulphonylureas or metformin but are superior to the former in reducing postprandial hyperglycaemia [25]. The complementary action of $\alpha$-glucosidase inhibitors with sulphonylureas in controlling both fasting and postprandial glucose concentrations was confirmed in a multicentre trial when the addition of a $\alpha$-glucosidase inhibitor to tolbutamide enhanced efficacy while attenuating undesirable effects on body weight and postprandial insulin [21]. Thus, acarbose plus tolbutamide reduced $\mathrm{HbA}_{1 \mathrm{c}}$ by $0.39 \%$ more than tolbutamide alone $(p<0.01)$.

Like the sulphonylureas, monotherapy with metformin also has a high secondary failure rate $(5-10 \%$ per year) [26] as the metabolic defects in Type 2 diabetes become more pronounced over time. Its complementary mode of action with acarbose led to their use as combination therapy. The addition of acarbose to the therapeutic regimen in patients inadequately controlled with metformin and diet lowered $\mathrm{HbA}_{1 \mathrm{c}}$, fasting and postprandial glucose as well as plasma insulin concentrations [22]. However, gastrointestinal side effects, including bloating, abdominal discomfort, diarrhoea and flatulence, which occur in up to $30 \%$ of patients treated with acarbose, frequently result in discontinuation of therapy. In one study, $56 \%$ of patients treated with acarbose plus metformin experienced gastrointestinal side effects and $12 \%$ discontinued therapy [22]. Recently, a second-generation $\alpha$-glucosidase inhibitor, miglitol, has been shown to have similar effects when combined with metformin [27], although the results are less impressive than those seen with a combination of metformin with insulin secretion enhancers.

The thiazolidinediones, rosiglitazone and pioglitazone, act by binding and activating the peroxisome proliferation-activated receptor- $\gamma$ (PPAR- $\gamma$ ) and, unlike sulphonylureas, do not stimulate insulin release or cause hypoglycaemia [18]. These agents can reduce mean $\mathrm{HbA}_{1 \mathrm{c}}$ both as monotherapy and in combination. In one study, rosiglitazone monotherapy dose-dependently reduced mean $\mathrm{HbA}_{1 \mathrm{c}}$ by $0.8 \%$ and $1.1 \%$ at doses of 4 and $8 \mathrm{mg}$ once daily, respectively, compared with placebo [28]. In combination with metformin, it improved glycaemic control, insulin sensitivity and beta-cell function. Mean $\mathrm{HbA}_{1 \mathrm{c}}$ concentrations decreased in patients treated with rosiglitazone combined with metformin $2.5 \mathrm{~g} /$ day $(1.0 \%$ and $1.2 \%$ decrease with 4 and $8 \mathrm{mg} /$ day) when compared with metformin plus placebo $(p<0.001)$ [29]. In parallel, a dose-dependent increase in body weight was reported. Such weight gain associated with glitazone treatment 
seems to be restricted to subcutaneous and not to visceral fat accumulation.

Pioglitazone in combination with metformin or sulphonylurea improved $\mathrm{HbA}_{1 \mathrm{c}}$ and FPG concentrations and produced beneficial effects on serum lipid concentrations $[30,31]$. Patients receiving pioglitazone (30 $\mathrm{mg}$ /day) plus metformin had a mean decrease in $\mathrm{HbA}_{1 \mathrm{c}}(0.83 \%$ decrease $)$ and FPG concentrations (37.7 mg/dl decrease) compared with metformin plus placebo $(p \leq 0.05)$ [30]. Similar effects were observed with pioglitazone in combination with a sulphonylurea - compared with placebo, $\mathrm{HbA}_{1 \mathrm{c}}$ decreased by $0.9 \%$ in patients receiving pioglitazone $15 \mathrm{mg}$ /day and $1.3 \%$ with pioglitazone $30 \mathrm{mg} /$ day [31]. Further investigations are required to determine the long-term effects of these combinations.

Combination therapy can postpone the use of insulin but as the condition progresses it is usually eventually required to restore glycaemic control. If glycaemic control cannot be attained with oral combination therapy, the use of insulin, either alone or in combination with low dose oral agents, should be considered without hesitation. The efficacy of bedtime intermediate-acting insulin in combination with sulphonylurea or metformin is well documented. The combination provides a well tolerated and effective method of normalising hyperglycaemia while minimising weight gain and postponing the need for multiple injections. One study has reported that bedtime or morning insulin induces less weight gain and hyperinsulinaemia than multiple injections, while achieving the same level of glycaemic control [23]. Likewise, the long-term use of bedtime intermediate-acting insulin plus metformin gave better glycaemic control, prevented weight gain and induced less hypoglycaemia $(1.8 \pm 0.4$ episodes per patient) than bedtime insulin plus sulphonylurea (3.4 \pm 1.0$)$, bedtime insulin plus sulphonylurea and metformin $(3.3 \pm 1.6)$, or bedtime and morning in-

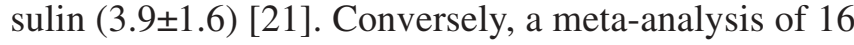
studies showed that combination therapy with insulin and sulphonylurea improves glycaemic control and lowers daily insulin dosage without adversely affecting body weight [32].

Although there are numerous treatment options, patients with Type 2 diabetes still have unmet medical needs. The reduction in efficacy over time with existing therapies and the emergence of increased postprandial glucose as an independent risk factor for vascular complications have fuelled the search for new antidiabetic medications.

\section{New approaches to combination therapy}

Restoring early-phase insulin secretion to reduce postprandial glucose excursions and prevent long-term hyperinsulinaemia is an important goal of therapy. Currently available insulin-secretion enhancers such as sulphonylureas do not have immediate effects on mealtime glucose excursions because of their slow onset of action (slow rate of absorption). Furthermore, their long duration of action confers a significant risk of hypoglycaemia unless used at low doses. For the same reasons, insulin sensitizers such as metformin do not adequately control postprandial glucose concentrations although there is some evidence showing that short-term rosiglitazone monotherapy can lower both fasting and postprandial glucose [33].

Nateglinide and repaglinide are new, rapid-onset, short-acting insulin-secretion enhancers. These agents act directly on pancreatic beta cells to stimulate insulin release and reduce postprandial hyperglycaemia [34, $35,36,37,38]$. Their mode of action is complementary to agents that target insulin resistance. Repaglinide, whose action is akin to that of a short-acting sulphonylurea, when used in combination with metformin results in additive glycaemic control compared with either agent alone, reducing $\mathrm{HbA}_{1 \mathrm{c}}$ from $8.3 \%$ at baseline to $6.9 \%$ [35]. At the end of the study, 59\% of patients receiving combination therapy with repaglinide and metformin achieved optimal $\mathrm{HbA}_{1 \mathrm{c}}$ concentrations of less than $7.1 \%$. Similarly, in a randomised study of 685 Type 2 diabetic patients inadequately controlled with diet and exercise, nateglinide plus metformin additively reduced $\mathrm{HbA}_{1 \mathrm{c}}$ by $1.5 \%$ from a baseline of $8.4 \%$ [36]. Here, more than $60 \%$ of the patients receiving combination therapy achieved $\mathrm{HbA}_{1 \mathrm{c}}$ values of less than $7 \%$, at the endpoint of the study (Fig. 2). The additive effects of nateglinide and metformin in combination were further confirmed in a trial of 467 patients with inadequate glycaemic control, after dietary intervention $\left(\mathrm{HbA}_{1 \mathrm{c}} 6.8-11 \%\right)$ and despite receiving maximal metformin dosages ( $\geq 1.5 \mathrm{~g} /$ day) [37]. Nateglinide (60 or $120 \mathrm{mg}$ before main meals) dose-dependently reduced $\mathrm{HbA}_{1 \mathrm{c}}$ with the greatest reduction being observed in patients with the highest baseline values of $\mathrm{HbA}_{1 \mathrm{c}}$ (Fig. 3). The efficacy of combination therapy with repaglinide or nateglinide plus metformin seems to be at least comparable to the traditional combination regimens described earlier. However, no clinical studies directly comparing outcomes with new versus traditional combination regimens have been reported to date.

Given their mechanism of action, it is feasible that nateglinide or repaglinide would be more effective when used in combination with, for example, an insulin sensitizer. The thiazolidinediones, rosiglitazone and pioglitazone, do not stimulate insulin release or cause hypoglycaemia [18] making them potentially suitable candidates for combination therapy with insulin-secretion enhancers. Although troglitazone is no longer available, a combination study of troglitazone with nateglinide [38] and a similar study with repaglinide [39] both showed a reduction of $\mathrm{HbA}_{1 \mathrm{c}}$ of $1.7 \%$ which was greater than the reduction seen with the respective monotherapies in the same trials. Ongoing studies with newer, actually available glitazones 


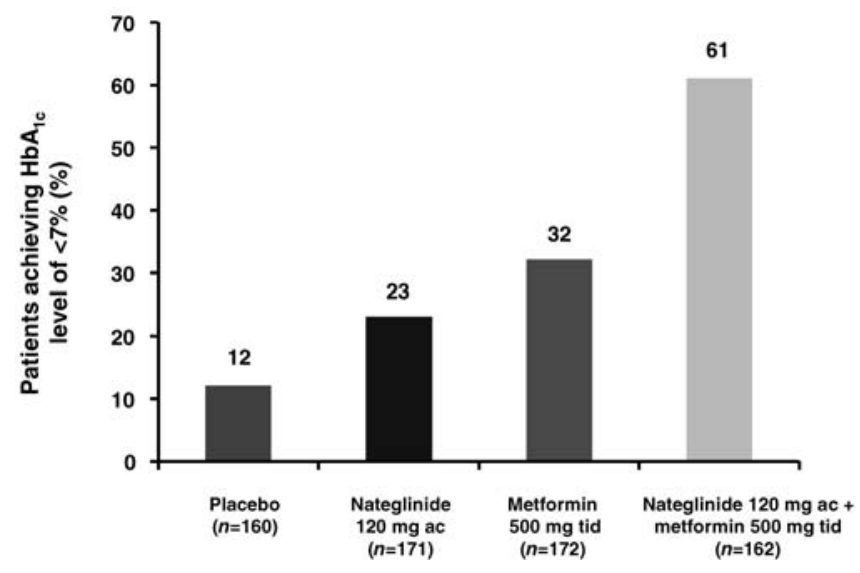

Fig. 2. Patients (\%) achieving an $\mathrm{HbA}_{1 \mathrm{c}}$ value of $<7 \%$ with placebo, nateglinide and metformin alone and in combination (intent-to-treat population)

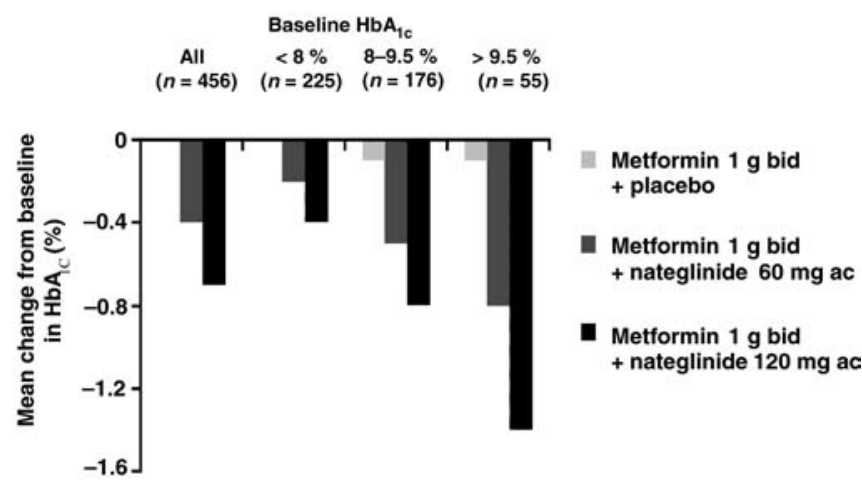

Fig. 3. Efficacy of nateglinide and metformin combination increased with increased severity of disease

will show if these previous effects are indicative of class action.

Similarly, insulin-secretion enhancers combined with $\alpha$-glucosidase inhibitors should be effective in controlling postprandial hyperglycaemia. Trials investigating nateglinide plus acarbose are currently underway while others with repaglinide combined with voglibose are planned. The combination of nateglinide or repaglinide and sulphonylureas, however, is not recommended as the 'fast on-fast off' mode of action of nateglinide and repaglinide is negated by the sustained action of sulphonylureas [40].

Adding repaglinide to NPH insulin monotherapy has recently been shown to be a possible combination therapy for patients with Type 2 diabetes, still providing a secretory reserve for insulin release [41].

\section{Safety and tolerability of oral antidiabetic agents}

Hypoglycaemia, weight gain, adverse effects on lipid profiles, hepatotoxicity, gastrointestinal symptoms and poor compliance are the main factors limiting the use of antidiabetic agents. Of the new insulin-secretion enhancers, repaglinide is associated with a greater risk of hypoglycaemia due to its slowly reversible mode of action. Symptomatic hypoglycaemia was more common in patients receiving combination therapy with repaglinide and metformin (33\%) compared with $10.7 \%$ and $0 \%$, respectively, than with repaglinide and metformin alone. However, the majority of episodes were mild and most were reported either during titration or by one particular subject [35]. Clinical trial data indicate that nateglinide in combination seems to have a low hypoglycaemic potential; symptoms suggestive of hypoglycaemia were reported in $26 \%$ of patients receiving nateglinide and metformin in combination [36]. However, clear comparisons across different studies are difficult to make unless identical criteria are used to define and detect hypoglycaemia.

Weight gain with antidiabetic therapy is influenced by the dose, duration and efficacy of treatment. This applies to insulin secretagogues at large and also to glycerides, although their associated weight gain is minimal and reaches less than $1 \mathrm{~kg}$ over a 6-month study period in combination with metformin [36]. There was also no evidence of an increase in liver enzymes, atherogenic changes in plasma lipids or interaction with commonly prescribed medications [36, 42].

\section{Conclusions}

Type 2 diabetes is a common, progressive disorder with devastating medical and social consequences [43]. There is a substantial body of evidence showing that the combination of oral antidiabetic agents with complementary mechanisms of action is highly effective in achieving and maintaining tight glycaemic control. Any effective combination therapy should improve clinical symptoms, quality of life, restore euglycaemia both in the fasting and postprandial state without inducing hypoglycaemia, and prevent or delay the development of long-term vascular complications.

Due to their short mode of action, insulin secretagogues such as repaglinide and nateglinide add new options for the management of Type 2 diabetes. The high secondary failure rate of antidiabetic monotherapy, regardless of the drug chosen, could indicate early combination therapy as a way towards better metabolic control in diabetic patients. Candidates for such early combination therapy with low doses of two oral agents, including a short acting insulin release, could be patients with $\mathrm{HbA}_{1 \mathrm{c}}$ above 8 or $9 \%$. Such an approach could be particularly suitable in elderly Type 2 diabetic patients. Any combination therapy should be based on an understanding of the natural history and pathophysiology of Type 2 diabetes, and the complementary pharmacological action of the antidiabetic agents used.

Acknowledgements. This work has been carried out with the help of a grant from Novartis Pharma AG, Basel, Switzerland. 


\section{References}

1. Avignon A, Radauceanu A, Monnier L (1997) Nonfasting plasma glucose is a better marker of diabetic control than fasting plasma glucose in Type 2 diabetes. Diabetes Care 20:1822-1826

2. Polonsky KS, Given BD, Hirsch LJ et al. (1988) Abnormal patterns of insulin secretion in non-insulin-dependent diabetes mellitus. N Engl J Med 318:231-239

3. Evans AJ, Krenz AJ (2001) Insulin resistance and $\beta$-cell dysfunction as therapeutic targets in type 2 diabetes. Diabetes Obes Metab 3:219-229

4. Monnier L (2000) Is postprandial glucose a neglected cardiovascular risk factor in Type 2 diabetes? Eur J Clin Invest 30 [Suppl 2]:3-11

5. Bruce DG, Chisholm DJ, Storlien LH, Kraegen EW (1988) Physiological importance of deficiency in early prandial insulin secretion in non-insulin-dependent diabetes. Diabetes 37:736-744

6. The DCCT Research Group (1995) The relationship of glycemic exposure $\left(\mathrm{HbA}_{1 \mathrm{c}}\right)$ to the risk of development and progression of retinopathy in the Diabetes Control and Complications Trial. Diabetes 44:968-983

7. UKPDS 38 (1998) Tight blood pressure control and risk of macrovascular and microvascular complications in Type 2 diabetes. (UKPDS 38). UK Prospective Diabetes Study (UKPDS) Group. BMJ 317:703-713

8. The DECODE study group on behalf of the European Diabetes Epidemiology Group (1999) Glucose tolerance and mortality: comparison of WHO and American Diabetes Association diagnostic criteria. Lancet 354:617-621

9. Okhubo Y, Kishikawa H, Araki E et al. (1995) Intensive insulin therapy prevents the progression of diabetic microvascular complications in Japanese patients with non-insulin dependent diabetes mellitus: a randomized prospective 6-year study. Diabetes Res Clin Pract 28:103-117

10. Ceriello A (2000) The postprandial state and cardiovascular disease: relevance to diabetes mellitus. Diabetes Metab Res Rev 16:125-132

11. Temelkova-Kurktschiev TS (2000) Postchallenge plasma glucose and glycemic spikes are more strongly associated with atherosclerosis than fasting glucose or $\mathrm{HbA}_{1 \mathrm{c}}$ level. Diabetes Care 23:1830-1834

12. Hanefeld M, Fischer S, Julius U et al. The DIS Group (1996) Risk factors for myocardial infarction and death in newly detected NIDDM: the Diabetes Intervention Study, 11-year follow-up. Diabetologia 39:1577-1583

13. Khaw K-T, Wareham N, Luben R et al. (2001) Glycated haemoglobin, diabetes and mortality in men in Norfolk cohort of European prospective investigation of cancer and nutrition (EPIC-Norfolk). BMJ 322:1-6

14. Turner RC, Cull CA, Frighi V, Holman RR (1999) Glycemic control with diet, sulphonylurea, metformin, or insulin in patients with Type 2 diabetes mellitus: progressive requirement for multiple therapies (UKPDS 49). UK Prospective Diabetes Study (UKPDS) Group. JAMA 281: 2005-2012

15. UKPDS 28 (1998) A randomized trial of efficacy of early addition of metformin in sulphonylurea-treated Type 2 diabetes. UK Prospective Diabetes Study (UKPDS) Group. Diabetes Care 21:87-92

16. UKPDS 33 (1998) Intensive blood glucose control with sulphonylureas or insulin compared with conventional treatment and risk of complications in patients with Type 2 diabetes. UK Prospective Diabetes Study (UKPDS) Group. Lancet 352:837-853
17. UKPDS 34 (1998) Effect of intensive blood-glucose control with metformin on complications in overweight patients with Type 2 diabetes (UKPDS 34). UK Prospective Diabetes Study (UKPDS) Group. Lancet 352:354-365

18. Riddle M (2000) Combining sulphonylureas and other oral agents. Am J Med 108:15S-22S

19. Inzucchi SE (2002) Oral antihyperglycemic therapy for type 2 diabetes. Scientific review. JAMA 287:360-372

20. Hermann LS, Schersten B, Bitzen PO et al. (1994) Therapeutic comparison of metformin and sulfonylurea, alone and in various combinations. A double-blind controlled study. Diabetes Care: 17:1100-1109

21. Coniff RF, Shapiro JA, Seaton TB, Bray GA (1995) Multicentre, placebo controlled trial comparing acarbose (BAY g 5421) with placebo, tolbutamide and tolbutamide plus acarbose in non-insulin dependent diabetes mellitus. Am J Med 98:443-451

22. Rosenstock J, Brown A, Fischer J, Jain A, Littlejohn T, Nadeau D (1998) Efficacy and safety of acarbose in metformin-treated patients with Type 2 diabetes. Diabetes Care 21:2050-2055

23. Yki-Järvinen H, Kauppila M, Kujansuu E, Lahti J, Marjanen T, Niskanen L (1992) Comparison of insulin regimens in patients with non-insulin dependent diabetes mellitus. N Engl J Med 327:1426-1433

24. Yki-Järvinen H, Ryysy L, Nikkila K, Tulokas T, Vanamo R, Heikkila M (1999) Comparison of bedtime insulin regimens in patients with Type 2 diabetes mellitus. A randomised, controlled trial. Ann Intern Med 130:389-396

25. Johnston PS, Lebovitz HE, Coniff RF et al. (1998) Advantages of alpha-glucosidase inhibition as monotherapy in elderly Type 2 diabetic patients. J Clin Endocrinol Metab 83:1515-1522

26. Hermann LS (1979) Metformin: a review of its pharmacological properties and therapeutic use. Diabetes Metab 5233-5245

27. Van Gaal L, Maislos M, Schernthaner G, Rybka J, Segal P (2001) Miglitol combined with metformin improves glycaemic control in Type 2 diabetes. Diabetes Obes Metab 3:326-331

28. Phillips LS, Grunberger G, Miller E, Patwardhan R, Rappaport EB, Salzman A, The Rosiglitazone Clinical Trials Study Group (2001) Once- and twice-daily dosing with rosiglitazone improves glycemia control in patients with type 2 diabetes. Diabetes Care 24:308-315

29. Fonseca V, Rosenstock J, Patwardhan R, Salzman A (2000) Effect of metformin and rosiglitazone combination therapy in patients with Type 2 diabetes mellitus: a randomised controlled trial. JAMA 283:1695-1702

30. Einhorn D, Rendell M, Rosenzweig J, Egan JW, Mathisen AL, Schneider RL (2000) Pioglitazone hydrochloride in combination with metformin in the treatment of type 2 diabetes mellitus: a randomized, placebo-controlled study, The Pioglitazone 027 Study Group. Clin Ther 22:1395-1409

31. Kipnes MS, Krosnick A, Rendell MS, Egan JW, Mathisen AL, Schneider RL (2001) Pioglitazone hydrochloride in combination with sulfonylurea therapy improves glycemic control in patients with type 2 diabetes mellitus: a randomized, placebo-controlled trial. Am J Med 111:10-17

32. Johnson JL, Wolf SL, Kabadi UM (1996) Efficacy of insulin and sulphonylurea combination therapy in Type 2 diabetes. A meta-analysis of the randomised placebo-controlled trials. Arch Intern Med 156:259-264

33. Raskin P, Rappaport EB, Cole ST, Yan Y, Patwardhan R, Freed MI (2000) Rosiglitazone short-term monotherapy lowers fasting and postprandial glucose in patients with Type 2 diabetes. Diabetologia 43:278-284 
34. Hanefeld M, Bouter KP, Dickinson S, Guitard C (2000) Rapid and short-acting mealtime insulin secretion with nateglinide controls both prandial and mean glycemia. Diabetes Care 23:202-207

35. Moses R, Slobodniuk R, Boyages S et al. (1999) Effect of repaglinide addition to metformin monotherapy on glycemic control in patients with Type 2 diabetes. Diabetes Care 22:119-124

36. Horton E, Clinkingbeard C, Gatlin M, Foley J, Mallows S, Shen S (2000) Nateglinide alone and in combination with metformin improves glycemic control by reducing mealtime glucose spikes in Type 2 diabetes. Diabetes Care 23:1660-1665

37. Marre M, Van Gaal L, Usadel K-H, Ball M, Whatmough I, Guitard C (2002) Nateglinide improves glycaemic control when added to metformin monotherapy: results of a randomised trial with type 2 diabetes patients. Diabetes Obes Metab 4:177-186
38. Rosenstock J, Gatlin M, Mallows S, Bush C (2000) Nateglinide improves glycemic control alone and in combination with troglitazone in patients with Type 2 diabetes. Diabetes 49 [Suppl 1]:498

39. Raskin P, Jovanovic L, Berger S et al. (2000) Repaglinide/troglitazone combination therapy: improved glycemic control in type 2 diabetes. Diabetes Care 23:979-983

40. Van Gaal L, Van Acker K, De Leeuw IH (2001) Repaglinide improves blood glucose control in sulphonylurea-naïve type 2 diabetes. Diabetes Res Clin Pract 53:141-148

41. De Luis DA, Aller R, Cuellar L et al. (2001) Effect of repaglinide addition to NPH insulin monotherapy on glycemic control in patients with type 2 diabetes. Diabetes Care 24: 1844-1845

42. Seltzer HS (1989) Drug-induced hypoglycaemia: a review of 1418 cases. Endocr Metab Clin North Am 18:163-183

43. Kalbag JB, Walter YH, Nedelman JR, McLeod JF (2001) Mealtime glucose regulation with nateglinide in healthy volunteers: comparison with repaglinide and placebo. Diabetes Care 24:73-77 